\title{
Effect of educational intervention using the
} Internet on quantitative ultrasound parameters in prevention of osteoporosis: a randomized
controlled trial in young Japanese women

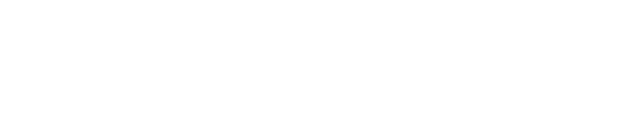

\author{
Kazumi Asakawa' \\ Katsuhiro Koyama ${ }^{2}$ \\ Zentaro Yamagata ${ }^{3}$ \\ 'Department of Fundamental \\ and Clinical Nursing, ${ }^{2}$ Department \\ of Health and Physical Education, \\ ${ }^{3}$ Department of Health Science, \\ University of Yamanashi, Chuo, \\ Yamanashi, Japan
}

\begin{abstract}
Background: The objective of this study was to determine whether or not educational intervention using the Internet, to prevent osteoporosis, is able to increase bone strength in young women.

Methods: Subjects were 253 healthy female university and junior college students aged 18-25 years. After initial measurements of bone stiffness index, a bone formation marker, and a bone absorption marker, the minimization method was used to allocate the subjects to an intervention group $(n=126)$ or a control group $(n=127)$ according to whether the measurements were above or below average. Subjects in the intervention group were instructed to perform osteoporosis prevention activities, ie, jump on the spot as high as possible ten times per day and increase calcium intake by $300 \mathrm{mg}$ per day to a total of $800 \mathrm{mg}$ or more per day on average. In addition, they were instructed to report the implementation status of the recommended measures via email. The researcher sent out information on osteoporosis and preventive behaviors to the subjects five times via email.
\end{abstract}

Results: A total of 182 subjects, comprising 87 (69.0\%) in the intervention group and 95 $(74.8 \%)$ in the control group, underwent remeasurement 6 months later. Of the subjects in the intervention group, $54(42.9 \%)$ reported their daily additional calcium intake amount and number of jumps via email. The mean amount of additional calcium taken was $216.3 \pm 85.9 \mathrm{mg}$ per day, and mean number of jumps performed was $6.4 \pm 4.2$ per day. Subjects in the intervention group were further divided into an implementation group $(n=54)$, consisting of subjects who sent in reports and a nonimplementation group $(n=72)$ who did not. No significant difference was found among the groups for rate of change in bone stiffness index and speed of sound, but there was a significant difference for broadband ultrasound attenuation (BUA) $(P=0.017)$. Sheffe's multiple comparison test was performed using baseline body weight and BUA values as covariates, and revealed that the rate of decrease in bone strength in the control group was larger than that in the implementation group $(P=0.049)$.

Conclusion: Health education for preventing osteoporosis via Internet email was performed over 6 months for women aged 18 to 25 and a comparison was performed between the intervention group and control group. The intervention consisted of high jumps on the spot (ten times a day), which reduced the drop in BUA, and thus indicates robustness of the trabecular structure. This suggests that a longer intervention period may maintain or improve bone strength.

Keywords: prevention, osteoporosis, Internet, ultrasound, Japanese, women

\section{Introduction}

Osteoporosis is a disorder that currently affects an estimated 75 million patients in Europe, Japan, and the US. ${ }^{1}$ It is important that we take preventive measures against osteoporosis 
not only in these countries, where populations are aging, but in Asian and African countries where populations are expected to age in the future. It has been shown that high-impact exercise and increased calcium intake are effective for the acquisition of peak bone mass in childhood, as well as for reducing bone loss and fracture risk during and after menopause. ${ }^{2,3}$

Daily calcium and vegetable intake is lowest in adult women in their 20s. ${ }^{4}$ The proportion of women in their $20 \mathrm{~s}$ with a low body mass index $(<18.5)$ is $25 \%$, which is the highest percentage of all the age groups. Without intervention, the number of postmenopausal patients seen with osteoporosis is expected to increase. Enhancing bone strength by health education may be effective in preventing osteoporosis among young women.

It has been reported that intervention via email is effective in promoting behavioral modification. ${ }^{5}$ However, there have been no reports that clearly show the effects of Internet education on behaviors to prevent osteoporosis. In this study, we provided health support to women aged 18-25 years (the age group when peak bone mass is acquired and maintained) via email with the aim of promoting a better understanding of osteoporosis and implementing preventive behaviors. The objective was to determine whether or not educational intervention using email, a commonly used communication tool, would increase bone strength in young women.

\section{Methods}

Subjects in this study were 253 healthy female students aged 18-25 years from the University of Yamanashi and Yamanashi Prefectural Junior Nursing College. The trial was carried out from May 2005 to January 2006. Subjects were given an adequate verbal explanation of the trial, and gave their written informed consent for voluntary participation. The study procedures were implemented according to the ethical guidelines for epidemiologic research and clinical studies, and approval was given by the ethics committee of the Faculty of Medicine, University of Yamanashi. On the basis of initial measurements, the subjects were stratified according to bone strength (stiffness index), a bone formation marker (bone alkaline phosphatase), and a marker of bone resorption (urinary N-telopeptide), and allocated to either an intervention group $(n=126)$ or a control group $(n=127)$.

\section{Educational program for intervention group}

The educational program involved setting targets for the amount of exercise and calcium intake required to prevent osteoporosis, ie, jumping on the spot as high as possible ten times a day and increasing calcium intake from dairy products by $300 \mathrm{mg} /$ day to a total of $800 \mathrm{mg} /$ day. Subjects were required to report the amount of calcium they had ingested and the number of jumps they had done on a weekly basis to the investigator by email. The investigator sent out information on osteoporosis and preventive behaviors to subjects via five emails, ie, foods containing a lot of calcium and amount of calcium contained in one serving of these foods (email 1), amount of calcium contained in milk, yogurt, and cheese by manufacturer and product (email 2), examples of ways of cooking to promote absorption of calcium into the body, eg, simultaneous intake of milk and lemon juice (email 3), types of exercise and their bone density-enhancing effects (email 4), and specific methods and advice for continuing the behaviors to prevent osteoporosis (email 5). Height and weight were measured at the start of the trial. A questionnaire survey on diet and exercise habits was carried out at the start of the trial and 6 months later.

\section{Instructions for control group}

After initial measurement of bone strength, each subject was told her measured bone density value and the average bone density of a 20-year-old woman. Each subject was also given a sheet explaining osteoporosis and preventive behaviors.

\section{Ultrasound bone parameter}

The quantitative ultrasound (QUS) method can be used to assess bone status easily without any exposure to radiation. Speed of sound (SOS) reflects bone density and the qualitative characteristics of bone, and broadband ultrasound attenuation (BUA) reflects the three-dimensional distribution of bone density and bone trabeculae. The bone stiffness index (SI) is used to evaluate SOS and BUA and is calculated as $0.67 \times \mathrm{BUA}+0.28 \times \mathrm{SOS}-420$. Bone mineral content and bone density values on imaging are currently used to diagnose osteoporosis. It has been demonstrated that there is a correlation between the SOS measured at the calcaneus by QUS and bone density at the lumbar spine measured using dual energy x-ray absorptiometry. ${ }^{6-12}$ The Achilles A-1000 Plus (Lunar Corporation, Madison, WI) can be used to measure SOS and BUA at the calcaneus, 95\% of which consists of cancellous bone. The ultrasound bone evaluation method provides information on the microstructure of bone and bone quality, and the calcaneus was used in this study 
because it is known to have a rapid metabolic turnover rate and is sensitive to the influence of a decrease in weightbearing. ${ }^{13-15}$

\section{Measurement of bone metabolism markers}

Bone maintains a balance between bone resorption and formation by exchanging information between osteoclasts and osteoblasts. Bone metabolism markers are thought to reflect changes in bone mass before and after measurement because they quantitatively indicate bone metabolism at the time of measurement. Bone metabolism markers are also indicators of bone quality, which is different from bone density. Type I collagen cross-linked N-telopeptide (NTX) is a bone resorption marker, in which NTX refers to fragments generated by the degradation of osteoclast collagen which are metabolized in the liver and kidney and excreted in urine. The test was carried out at approximately the same time of day, because this marker exhibits diurnal variation. The second urine sample was collected and measured. Bone alkaline phosphatase (BAP) is a bone formation marker in the serum that is produced at different stages of osteoblast differentiation. Measurement of these bone metabolism markers was outsourced to Mitsubishi Kagaku Bio-Clinical Laboratories Inc, Tokyo, Japan.

\section{Statistical analysis}

An unpaired $t$-test was used to compare the averages between the two groups. To compare the averages between the three groups, one-way analysis of variance was performed, followed by Sheffe's multiple comparisons test. Weight was used as a covariate in every comparison between the groups. Differences between percentages were assessed using a Chi-squared test with a significance level of 5\%. Statistical analysis software (SPSS 13.0J, SPSS Inc, Chicago, IL) was used.

\section{Results}

\section{Baseline measurements}

Tables 1 and 2 show the mean baseline values for the intervention and control groups. There were no significant differences between the groups in terms of age, weight, body mass index, bone strength, ie, SI, SOS, and BUA, or bone metabolism markers, ie, BAP and NTX (unpaired $t$-test; $P>0.05)$. There was also no difference between the two groups in terms of the percentages of subjects who had good exercise habits, ie, those who had exercised for at least 30 minutes at a time twice weekly or more (Chi-squared test, $P>0.05$, data not shown).
Table I Age and anthropometric measures

\begin{tabular}{lll}
\hline & $\begin{array}{l}\text { Intervention }(\mathbf{n}=\mathbf{I 2 6}) \\
(\mathbf{m e a n} \pm \mathbf{S D})\end{array}$ & $\begin{array}{l}\text { Control }(\mathbf{n}=\mathbf{I 2 7}) \\
(\text { mean } \pm \text { SD })\end{array}$ \\
\hline Age & $19.29 \pm 1.52$ & $19.31 \pm 1.38$ \\
Height $(\mathrm{cm})$ & $158.49 \pm 4.96$ & $159.02 \pm 5.42$ \\
Weight $(\mathrm{kg})$ & $52.03 \pm 8.18$ & $51.10 \pm 5.83$ \\
BMI & $20.55 \pm 3.62$ & $20.26 \pm 2.23$ \\
\hline
\end{tabular}

Note: $t$-test, $P>0.05$.

Abbreviations: BMI, body mass index; $n$, number; SD, standard deviation.

\section{Effects of intervention}

Of the 253 subjects, 182 (the intervention group $[\mathrm{n}=87,69.0 \%]$ and the control group $[\mathrm{n}=95,74.8 \%])$ underwent remeasurement 6 months after the start of the trial (Figure 1). Bone density and bone metabolism markers were compared between the intervention group and the control group. For subjects who were not remeasured after 6 months, baseline levels were used as the remeasurement values. Intention to treat analysis was performed to identify if the intervention had made any difference to bone strength.

There were no significant differences between the groups for bone strength parameters measured at baseline and 6 months later (Table 2). Rate of change was calculated by dividing the variations in SI, SOS, and BUA over 6 months by the baseline values. No significant difference was observed in the rate of change for SI and SOS between the groups. However, the control group showed a significantly higher rate of decline in BUA than the intervention group (unpaired $t$-test, $P=0.01$, Figure 2).

There were no significant differences between the groups for bone metabolism markers measured at baseline and 6 months later. The rate of change was calculated by dividing the variations in bone alkaline phosphatase and NTX over 6 months by the baseline values. BAP decreased, whereas NTX increased, but no significant differences were observed between the groups for both BAP and NTX.

How well the subjects had implemented the recommended behaviors to prevent osteoporosis during the previous 6 months was investigated using a questionnaire at remeasurement 6 months after the start of the trial. In both groups, the percentage of subjects who had increased their calcium intake was about $50 \%$, and the percentage of subjects who had done at least 30 minutes of exercise once a week or more was about $20 \%$ (Table 3). Sixty-four (50.8\%) of the intervention subjects and ten $(7.9 \%)$ of the controls had performed high jumps on the spot ten times a day, showing a significant difference between the two groups (Chi-squared test, $P<0.001)$. 


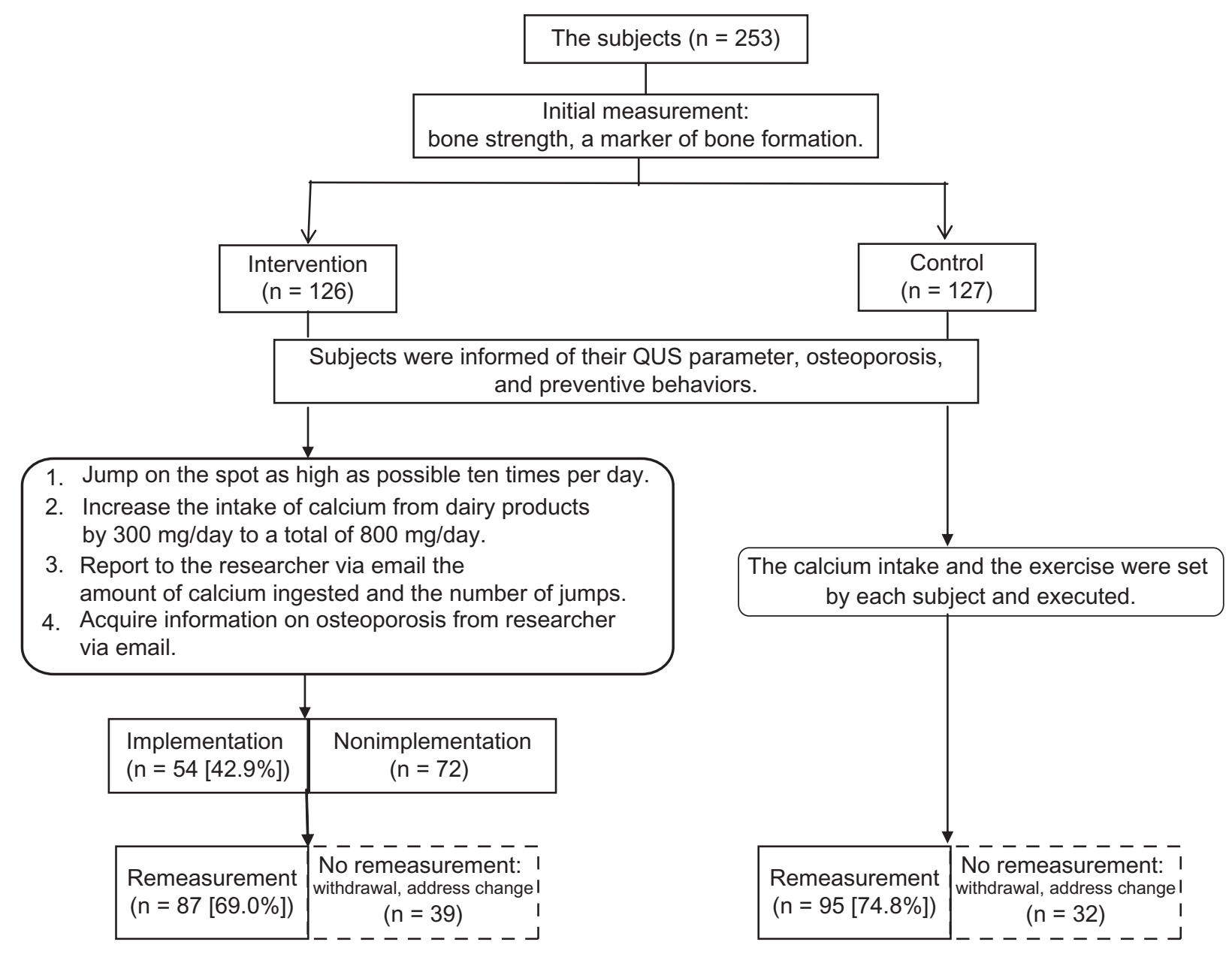

Figure I Progress of the study and subjects.

Abbreviations: n, number; QUS, quantitative ultrasound.

\section{Effects of jumping and increased calcium intake}

In the intervention group, 54 subjects (42.9\%) had reported their daily calcium intake and number of jumps per day via email. The average amount of additional calcium ingested each day, as calculated from the data sent to the researcher, was $216.3 \pm 85.9 \mathrm{mg}$, whereas the average number of jumps performed per day was $6.4 \pm 4.2$. Subjects in the intervention group were divided into two groups, ie, an implementation group, which consisted of the subjects who reported the number of jumps performed and the intake of calcium (in other words, those who implemented preventive behaviors for osteoporosis) and a nonimplementation group, which consisted of the subjects who did not send in reports. Comparison was conducted among these two groups and the control group in regard to changes in bone

Table 2 QUS parameters and bone metabolism markers at baseline and 6 months later

\begin{tabular}{|c|c|c|c|c|}
\hline & \multicolumn{2}{|c|}{ Intervention $(n=126)$} & \multicolumn{2}{|c|}{ Control $(n=127)$} \\
\hline & $\begin{array}{l}\text { Baseline } \\
(\text { mean } \pm \text { SD) }\end{array}$ & $\begin{array}{l}6 \text { months later } \\
(\text { mean } \pm S D)\end{array}$ & $\begin{array}{l}\text { Baseline } \\
(\text { mean } \pm \text { SD) }\end{array}$ & $\begin{array}{l}6 \text { months later } \\
\text { (mean } \pm \text { SD) }\end{array}$ \\
\hline SI & $92.68 \pm|2.8|$ & $94.83 \pm 12.65$ & $94.44 \pm 12.12$ & $95.83 \pm 12.40$ \\
\hline $\mathrm{SOS}(\mathrm{cm} / \mathrm{s})$ & $|563.6| \pm 28.0 \mid$ & $1573.36 \pm 28.52$ & $1566.80 \pm 26.83$ & $1576.48 \pm 26.75$ \\
\hline BUA & $111.63 \pm 10.48$ & $111.37 \pm 9.67$ & $113.83 \pm 9.10$ & $111.65 \pm 10.12$ \\
\hline BAP & $25.37 \pm 7.17$ & $22.80 \pm 6.57$ & $25.12 \pm 7.47$ & $22.99 \pm 7.37$ \\
\hline NTX & $66.41 \pm 32.03$ & $66.98 \pm 30.12$ & $66.98 \pm 30.12$ & $63.13 \pm 30.26$ \\
\hline
\end{tabular}

Abbreviations: QUS, quantitative ultrasound; n, number; SD, standard deviation; SI, stiffness index; SOS, speed of sound; BUA, broadband ultrasound attenuation; BAP, bone alkaline phosphatase; NTX, urinary n-telopeptide. 


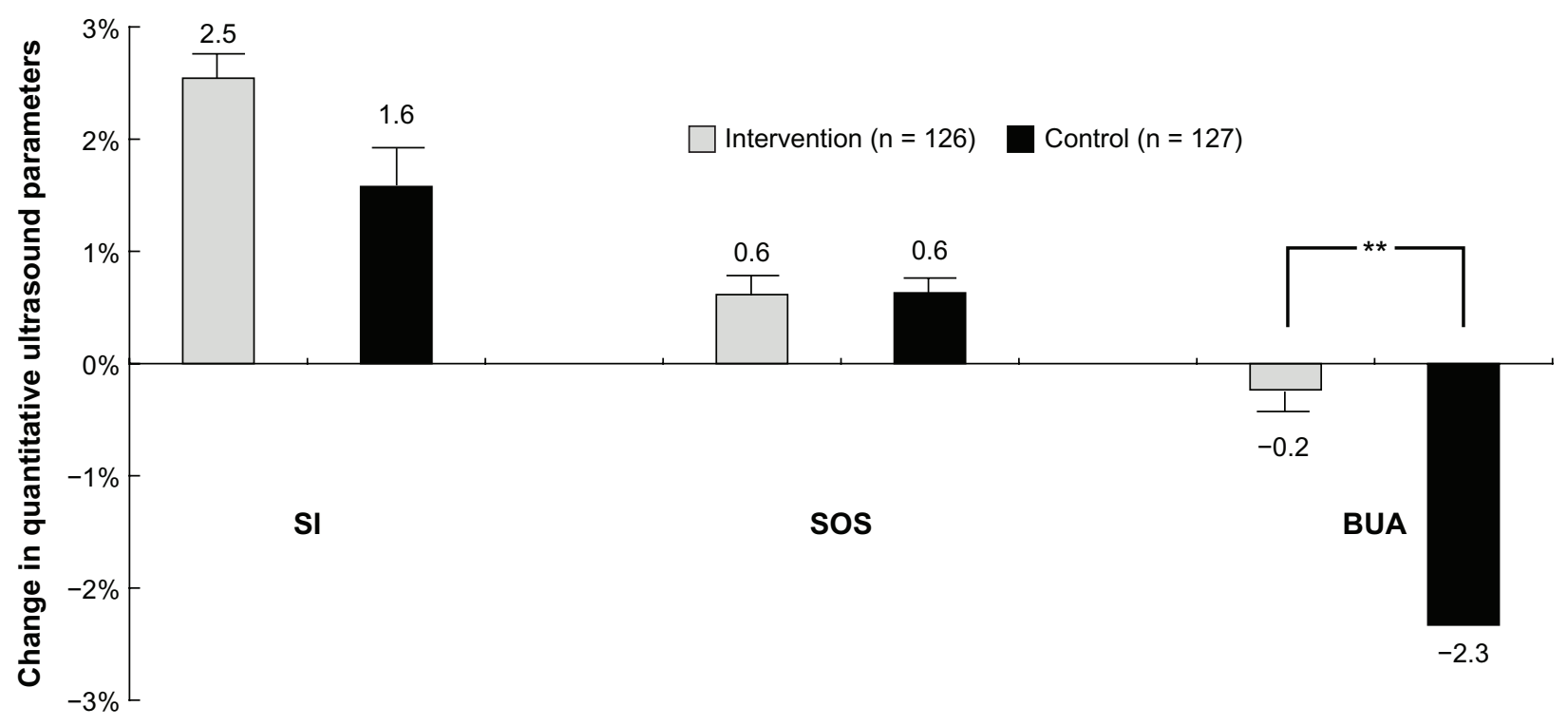

Figure 2 Change in quantitative ultrasound parameters of calcaneus in intervention group and control group. Note: $* *$ t-test, $P<0.01$.

Abbreviations: $n$, number; SI, stiffness index; SOS, speed of sound; BUA, broadband ultrasound attenuation.

strength parameters and changes in bone metabolism markers.

There were no significant differences among the implementation, nonimplementation, and control groups in any of the bone strength parameters or bone metabolism markers measured at the start of the trial and 6 months later (Figure 3). The rate of change was calculated by dividing variations during the 6 months by the initial measured values. Analysis of variance performed for the three groups showed no significant difference in the rate of change of bone SI and SOS, but there was a significant difference for BUA $(P=0.017)$. In addition, a multiple comparisons test was conducted using weight and the baseline BUA value as covariates. Sheffe's multiple comparison test showed that the rate of decrease in the control group was larger than that in the implementation group $(P=0.049)$. Each group showed a decrease in BAP, and the implementation group had an increased NTX. However, no statistically significant

Table 3 Execution ratio of osteoporosis prevention action for 6 months

\begin{tabular}{lll}
\hline & $\begin{array}{l}\text { Intervention } \\
(\mathbf{n}=126)\end{array}$ & $\begin{array}{l}\text { Control } \\
(\mathbf{n}=127)\end{array}$ \\
\hline Calcium intake increase & $66(52.3 \%)$ & $60(47.2 \%)$ \\
Exercise & $26(20.6 \%)$ & $29(22.8 \%)$ \\
Jump (ten times per day)** & $64(50.8 \%)$ & $10(7.9 \%)$ \\
\hline
\end{tabular}

Notes: Exercise; movement executed more than once per week for at least 30 minutes each time. ${ }^{* *} \chi^{2}, P<0.001$.

Abbreviation: $\mathrm{n}$, number. differences were observed among the implementation, nonimplementation, and control groups.

\section{Discussion}

This study was carried out using more than 100 subjects in each group in order to understand changes in bone status over a long period of time and evaluate the effects of intervention. For osteoporosis treatment, a minimum of $800 \mathrm{mg}$ of calcium intake per day is required. ${ }^{16}$ The average amount of calcium intake in Japanese women is $523 \mathrm{mg}$. Therefore, this study aimed to increase current calcium intake by $300 \mathrm{mg}$ /day to a total of at least $800 \mathrm{mg} /$ day. We considered dairy products to be foods that enable the body to absorb calcium most efficiently and enable people to take calcium by mouth in a continuous manner in order to increase the amount of calcium intake. However, because the actual amount of additional calcium intake was $216.3 \mathrm{mg}$ /day on average, the total amount of calcium intake per day was estimated at $739 \mathrm{mg}$ (an average intake of $523 \mathrm{mg}$ for women in their 20s plus $216 \mathrm{mg}$ ).

It is also reported that an increase in the amount of vitamin $\mathrm{D}$ and calcium intake decreases the risk of falls, resulting in decreased fragility fractures caused by falls among the elderly. ${ }^{17,18}$ Vitamin D promotes bone formation and plays a part in protein synthesis in muscles and muscle contraction. It is recommended that young women take $11 \mu \mathrm{g}$ (400 IU) of vitamin D per day. ${ }^{19}$ Young Japanese women take $6.5 \mu \mathrm{g}$ /day of vitamin $\mathrm{D}$ on average compared with 


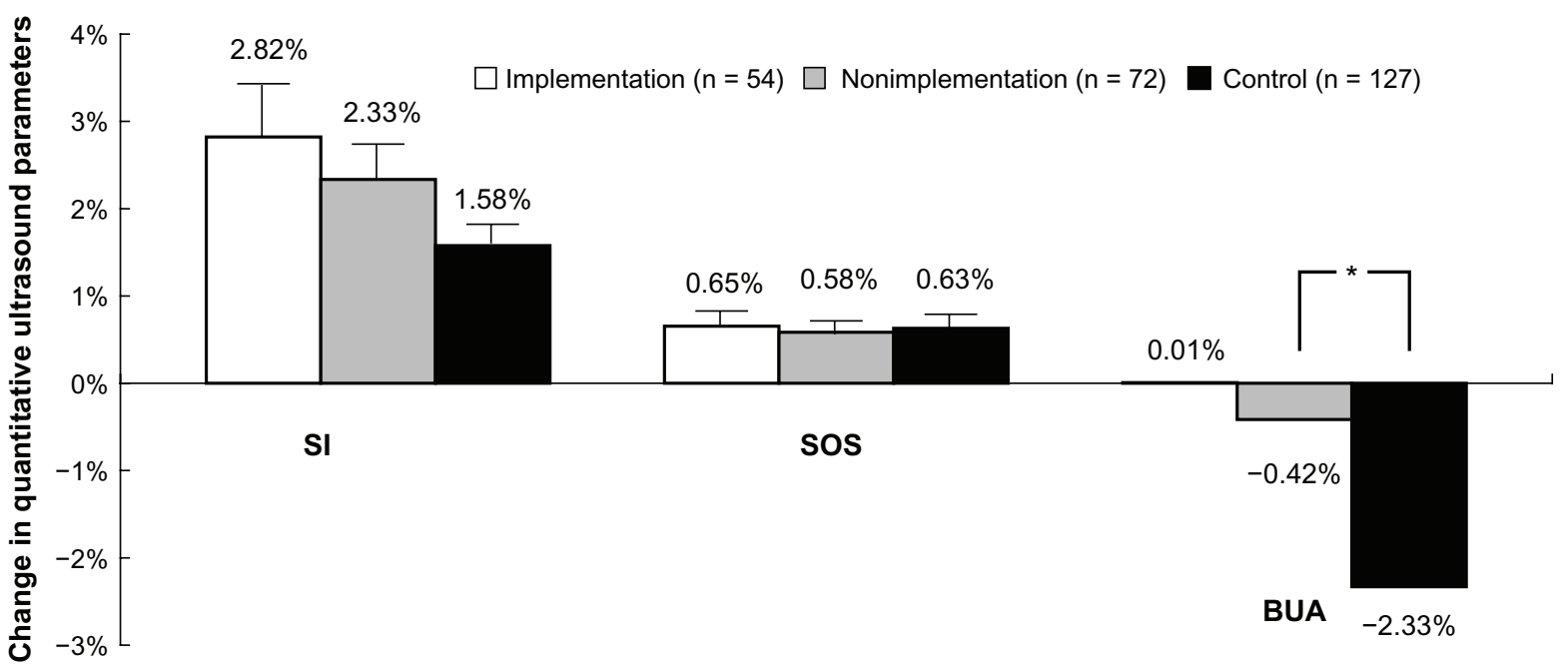

Figure 3 Change in quantitative ultrasound parameters of calcaneus in implementation, nonimplementation and control groups. Note: $* P<0.05$.

Abbreviations: SI: stiffness index; SOS, speed of sound; BUA, broadband ultrasound attenuation; n, number.

the nutrition intake standard of $5.5 \mu \mathrm{g} /$ day. ${ }^{20}$ Given that the average intake amount meets the intake standard, this study did not set a target for vitamin D ingestion.

This study set the target for weight-bearing exercise to ten jumps per day, but the actual number of jumps reported was 6.4 per day. Shibata et al reported that performing the jumping exercise over 1 year increased BAP for women in their $30 \mathrm{~s}^{2}$ However, the intervention period of the present study was shorter at 6 months, and this bone metabolism marker was in a period of change towards slow turnover, so there was significant intraindividual variation. In our opinion, that is why no effect of the intervention was observed. The most effective methods for maintaining adequate calcium, vitamin $\mathrm{D}$, and protein intake, as well as weight-bearing exercise must be considered, in the future.

Comparison of the rate of change in ultrasound bone density measurements over 6 months between the intervention group and control group showed no difference in SI, which represents overall bone strength. Both SOS and BUA reflect bone stiffness. ${ }^{21}$ SOS is determined by Young's modulus and bone density, which reflects the total mass of cortical bone and cancellous bone, and shows a high correlation with bone density. Meanwhile, BUA reflects the trabecular structure of cancellous bone. SOS and BUA values reflect different aspects of bone. ${ }^{22}$

SOS increased slightly at the same rate in both groups, whereas the BUA value in the control group decreased significantly compared with that in the intervention group. The measurement error of BUA with the same equipment was $0.9 \%-1.2 \%$ according to Yamazaki. ${ }^{23}$ Because the difference in the rate of change of BUA over 6 months between the intervention group and control group was $2.1 \%$, the rate of decrease in BUA is considered to have been reduced by the intervention over 6 months.

In this study the researcher measured bone strength at the calcaneus, $90 \%$ of which consists of cancellous bone. This site has a rapid metabolic turnover and is most susceptible to the influence of zero gravity and nonweight-bearing conditions. ${ }^{24-26}$ In a study of 181 people aged $14-17$ years, it was reported that BUA and SI had a high correlation with weight and body mass index, but had no relationship with SOS. It can be considered that the exercise of jumping as high as possible stimulated the trabecular structure of the calcaneus and reduced the decrease in BUA.

Mobile phone email is easy to use and convenient, and the user can view messages anytime and anywhere. This enabled a single researcher to determine the implementation status of a large number of subjects and communicate with the email senders. However, because it is easy to change email address using a mobile phone, there were some subjects with whom the researcher lost contact and could not continue the intervention to the end of the trial period.

An intention-to-treat analysis was used to evaluate insignificant effects when determining the results of a randomized controlled trial. In this study, BUA was decreasing after 6 months. It cannot be denied that the use of values measured at the start of trial for subjects who were not to be subjected to remeasurements 6 months 
later contributed to a reduction in the rate of decrease. However, the intention-to-treat analysis was carried out because there was no significant difference between the groups in the percentage of subjects who underwent remeasurement.

Young women have the lowest levels of nutritional intake, exercise, and health awareness of all the age groups, probably because they are at an age where they have almost no concern about health. The number of those who increased their calcium intake and those who had gained the habit of regular exercise had increased in the present study compared with the baseline. We consider that recognition of their bone strength by measurement raised their awareness of osteoporosis. At the same time, the knowledge that they obtained encouraged them to change their health behavior. Better understanding of osteoporosis and implementation of preventive behaviors should have a good influence on the child-rearing ability of these women, because their generation will become mothers in a few years. In this sense, the educational intervention was socially meaningful.

There are limitations to generalizing the results of this study because its subjects were limited to female university and junior college students and women who were in their 20 s and not selected at random. In addition, considering that the subjects were young women, this study used the noninvasive ultrasound method and measured the calcaneus, ie, peripheral bone. Given that bone density varies at different anatomical sites, the measurements in this study do not reflect increased bone mass in the whole body, particularly in the vertebral and femoral areas. It cannot be denied that the number of subjects was insufficient to evaluate the effects of intervention, because those who implemented osteoporosis preventive behaviors (and reported their calcium intake amount and number of jumps) accounted for $42 \%$ of all the subjects and those who underwent remeasurement accounted for approximately $70 \%$.

\section{Conclusion}

Health education for preventing osteoporosis using Internet email was carried out for women aged 18-25 years, but no significant difference in bone stiffness index, an indicator for bone strength, was observed between the intervention group and the control group. However, the intervention consisted of high jumps on the spot (ten times a day), which reduced the drop in BUA, and thus indicates robustness of the trabecular structure. This suggests that a longer intervention period may maintain or improve bone strength.

\section{Disclosure}

The authors report no conflicts of interest in this work.

\section{References}

1. Report of a WHO Scientific Group. WHO Scientific Group on Prevention and Management of Osteoporosis Prevention and management of osteoporosis; 2005.

2. Shibata Y, Ohsawa I, Watanabe T, Miura T, Sato Y. Effect of physical training on bone mineral density and bone metabolism. J Physiol Anthropol Appl Human Sci. 2003;22:203-208.

3. Heaney RP. Calcium, daily products, and osteoporosis. J Am Coll Nutr. 2000;2:83S-99S.

4. Health and Welfare Statistics Association. Handbook of Health and Welfare Statistics. Tokyo, Japan: Health and Welfare Statistics Association. 2011;58:48-52.

5. Bessell TL, McDonald S, Silagy CA, Anderson JN, Hiller JE, Sansom LN. Do Internet interventions for consumers cause more harm than good? a systematic review. Health Expect. 2002;5:28-37.

6. Ahuja SP, Greenspan SL, Lin Y, Bowen A, Bartels D, Goyal RK. A pilot study of heel ultrasound to screen for low bone mass in children with leukemia. J Pediatr Hematol Oncol. 2006;28:427-432.

7. Bauer DC, Ewing SK, Cauley JA, et al. Quantitative ultrasound predicts hip and non-spine fracture in men: the MrOS study. Osteoporos Int. 2007;18:771-777.

8. Díez-Pérez A, Marín F, Vila J, et al. Evaluation of calcaneal quantitative ultrasound in a primary care setting as a screening tool for osteoporosis in postmenopausal women. J Clin Densitom. 2003;6:237-245.

9. Greenspan SL, Bouxsein ML, Melton ME, et al. Precision and discriminatory ability of calcaneal bone assessment technologies. J Bone Miner Res. 1997;12:1303-1313.

10. López-Rodríguez F, Mezquita-Raya P, de Dios Luna J, EscobarJiménez F, Muñoz-Torres M. Performance of quantitative ultrasound in the discrimination of prevalent osteoporotic fractures in a bone metabolic unit. Bone. 2003;32:571-578.

11. Pinheiro MM, Castro CM, Szejnfeld VL. Low femoral bone mineral density and quantitative ultrasound are risk factors for new osteoporotic fracture and total and cardiovascular mortality: a 5-year populationbased study of Brazilian elderly women. J Gerontol A Biol Sci Med Sci. 2006;61:196-203.

12. Trimpou P, Bosaeus I, Bengtsson BA, Landin-Wilhelmsen K. High correlation between quantitative ultrasound and DXA during 7 years of follow-up. Eur J Radiol. 2010;73:360-364.

13. Vogel JM, Wasnich RD, Rossa PD. The clinical relevance of calcaneus bone mineral measurements: a review. Bone Miner. 1988;5:35-58.

14. Cummings SR, Black DM, Nevitt MC, et al. Bone density at various sites for prediction of hip fracture. Lancet. 1993;341:72-75.

15. Eeach H, Pietschmann P, Bernecker P, et al. Broadband ultrasound attention: a new diagnostic method in osteoporosis. Am J Roentgenol. 1990;155:825-828.

16. Orimo H. Japanese Guidelines for the Prevention and Treatment of Osteoporosis. Tokyo, Japan: Life Science Publishing Co, Ltd; 2006:16.

17. Avenell A, Gillespie WJ, Gillespie LD, et al. Vitamin D and vitamin D analogues for preventing fractures associated with involutional and post-menopausal osteoporosis. Cochrane Database Syst Rev. 2005;3: CD000227.

18. Bischoff-Ferrari HA, Willet WC, Wong JB, et al. Fracture prevention with Vitamin D supplementation. A meta-analysis of randomized controlled trials. JAMA. 2005;293:2257-2264.

19. Nakamura K. Vitamin D intake and the prevention of osteoporosis. Clin Calcium. 2005;5:1386-1391.

20. Ministry of Health, Labor and Welfare. Standard tables of food composition in Japan; 2010.

21. Sakaguchi S, Kusida K, Yamazaki K, Inoue T. Improvement of quantitative bone ultrasound. Osteoporosis Japan. 1996;4: $22-26$. 
22. Yamazaki K. Quantitative bone ultrasound and dynamic index of bone. Journal of Japanese Society of Bone Morphometry. 1996;6: $7-12$.

23. Yamazaki K. [Cross-calibration of bone volume assessment.] Nippon Rinsho. 2004;62:309-311. [Japanese.]
24. Vogel JM. Use of bone mineral measurements in the management of osteoporosis. West J Med. 1996;165:304-305.

25. Leblanc AD, Spector ER, Evans HJ, Sibonga JD. Skeletal responses to space flight and the bed rest analog: a review. J Musculoskelet Neuronal Interact. 2007;7:33-47.

\section{Publish your work in this journal}

The International Journal of Women's Health is an international, peerreviewed open-access journal publishing original research, reports, reviews and commentaries on all aspects of women's healthcare including gynecology, obstetrics, and breast cancer. Subject areas include: Chronic conditions (migraine headaches, arthritis, osteoporosis);
Endocrine and autoimmune syndromes; Sexual and reproductive health; Psychological and psychosocial conditions. The manuscript management system is completely online and includes a very quick and fair peer-review system. Visit http://www.dovepress.com/ testimonials.php to read real quotes from published authors.

Submit your manuscript here: http://www.dovepress.com/international-journal-of-womens-health-journal 